The Analogy between Hydraulic Jumps in Liquids and Shock Waves in Gases

F. R. Gilmore, M. S. Plesset, and H. E. Crossley, JR. 
Reprinted from Journal of Applied Phy sics, Vol. 21, No. 3, 243-249, March, 1950

Copyright 1950 by the American Institute of Physics

Printed in U. S. A.

\title{
The Analogy between Hydraulic Jumps in Liquids and Shock Waves in Gases*
}

\author{
F. R. Gilmore, M. S. Plesset, and H. E. Crossley, Jr. \\ Hydrodynamics Laboratory, California Institute of Technology, Pasadena, California
}

(Received September 30, 1949)

\begin{abstract}
The theory of the hydraulic jump is presented briefly, and the analogy between this phenomenon and the compression shock wave in gases is pointed out. The results of experimental measurements of hydraulicjump intersections on a water table are reported. Considerable disagreement between theory and experiment is found. Other investigators have noted a disagreement between theory and experiment for compressionshock intersections in gases. The discrepancy in the aerodynamic case appears unlike that found in the hydraulic case. Possible reasons for the discrepancy in the hydraulic case are discussed; some sources of error are peculiar to hydraulic jumps and do not apply to compression shocks. Such factors limit the utility of the water table as an analog device.
\end{abstract}

\section{INTRODUCTION}

$\mathbf{I}_{\mathrm{o}}^{\mathrm{T}}$ $\mathrm{T}$ has been known for a long time that liquid flow in open channels is of two different types: relatively smooth "streaming" flow at low speeds, and high speed "shooting" flow characterized by standing waves and frequently by sudden changes in depth known as hydraulic jumps. More recently, the increased study of compressible-flow phenomena has led investigators to note the resemblances between streaming channel flow and subsonic compressible flow, and between shooting channel flow and supersonic compressible flow. This similarity was put on a mathematical basis by Jouguet ${ }^{1}$ and Riabouchinsky. ${ }^{2}$ Further theoretical investigations have been carried out by von Kármán ${ }^{3}$ and by Preiswerk ${ }^{4}$; experimental studies have been made by Ippen $^{5}$ and by Binnie and Hooker. ${ }^{6}$ Later work ${ }^{7}$ has been concerned with the application of the theory to model testing, except for a recent theoretical paper by Stoker. ${ }^{8}$

* This study was supported by the ONR and the Navy Bureau of Ordnance.

${ }^{1}$ E. Jouguet, J. de math. pures et appliq. (Series 8) 3, 1 (1920).

2 D. Riabouchinsky, Comptes Rendus 195, 998 (1932); 199, 632 $(1934) ; 202,1725$ (1936).

3 Th. von Kármán, Zeits. f. angew. Math. Mech. 18, 49 (1938)

${ }^{4}$ E. Preiswerk, N.A.C.A. Tech. Memo. 934 and 935 (1940); translated from Mitteilungen Inst. f. Aerodyn., No. 7, Eidg. Tech. Hochsch., Zurich (1938).

${ }^{5}$ A. Ippen, Ph.D. thesis, California Institute of Technology (1936).

6 A. M. Binnie and S. G. Hooker, Proc. Roy. Soc. 159, 592 (1937).

${ }^{7}$ See, for example, Orlin, Lindner, and Bitterly, N.A.C.A. Report 875 or Tech. Note 1185 (1947).

${ }^{8}$ J. J. Stoker, Commun. App. Math. 1, 1 (1948).
The analogy between liquid flow with a free surface and two-dimensional gas flow has been found useful for qualitative investigations of high speed gas flow by means of relatively simple water-table installations. However, recent advances in aerodynamics have brought many problems to the point where more accurate information is required than can be obtained from the hydraulic analogy. The present utility of the water table as an analog device is limited to special problems in aerodynamics, such as transient phenomena in high speed flow, and shock-wave intersection phenomena, particularly of the Mach type.

The discussion here is concerned with the analogy between hydraulic-jump intersections on a water table and shock-wave intersections in gases.

\section{THEORY}

\section{Shock Waves in Compressible Gas Flow}

As is well known, a compression wave of finite amplitude in a gas grows continually steeper as the wave progresses until a finite limiting steepness determined by viscous and heat-conduction effects is attained. The limiting thickness of the shock in the usual situation is negligible compared with the other dimensions in the flow field. The flow relations across a straight compression shock are determined by the conservation laws for mass, momentum, and energy without consideration of the conditions inside the shock. The results are the 
familiar Rankine-Hugoniot equations. ${ }^{9}$ Figure 1 shows curves of the density ratio, temperature ratio, and the square root of the pressure ratio across a normal shock as functions of the initial Mach number for perfect gases having specific-heat ratios of $\gamma=1.4$ and $\gamma=2$.

The flow relations across a straight shock which is oblique to the flow may be obtained by superimposing on the normal velocity an additional velocity tangential to the shock which remains unchanged across the shock. For a curved shock, if the radius of curvature is much larger than the shock thickness, the flow relations may be obtained by approximating the curved shock with a number of straight segments.

\section{Hydraulic Jumps in Liquid Flow with a Free Surface}

If an elevation wave of finite amplitude is produced on the surface of a liquid, Stoker ${ }^{8}$ has shown from the equations of motion that the wave front will grow continually steeper as it progresses, until finally the wave leans forward. At this point experiments show that the wave form deviates from the theoretical form, with the formation of a breaker or "roller" and the establishment of a turbulent wave of constant shape (except for minor fluctuations of short period). Such a steady finite wave is called a hydraulic jump. Bakhmeteff and Matzke $^{10}$ have found experimentally that

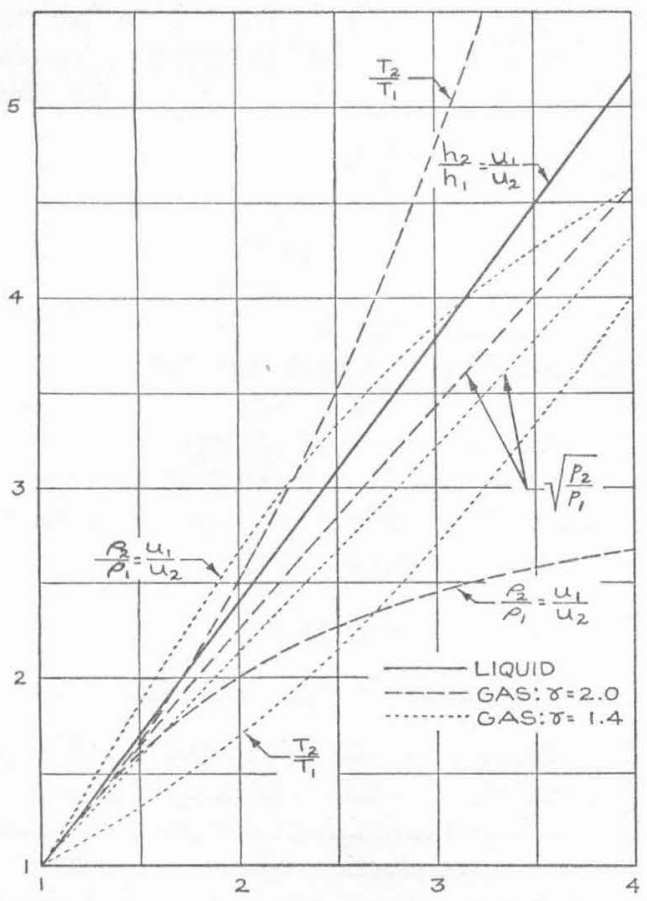

Fig. 1. Flow relations across a normal shock, or hydraulic jump. $\mathrm{M}_{1}=$ Mach number ahead of shock.

${ }^{9}$ See R. Courant and K. O. Friedrichs, Supersonic Flow and Shock Waves (Interscience Publishers, Inc., New York, 1948), pp. 116-125, or similar texts on compressible flow.

${ }^{10}$ B. Bakhmeteff and A. Matzke, Trans. A.S.C.E. 101, 630 (1936).

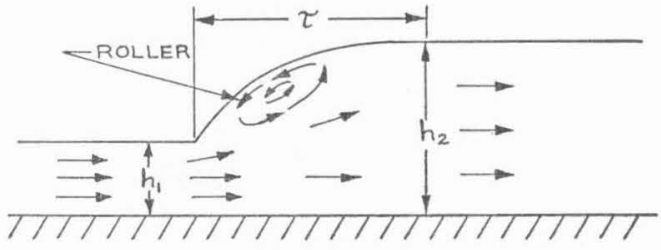

Fig. 2. Typical profile of a strong hydraulic jump $\left(h_{2} / h_{1}>2\right)$.

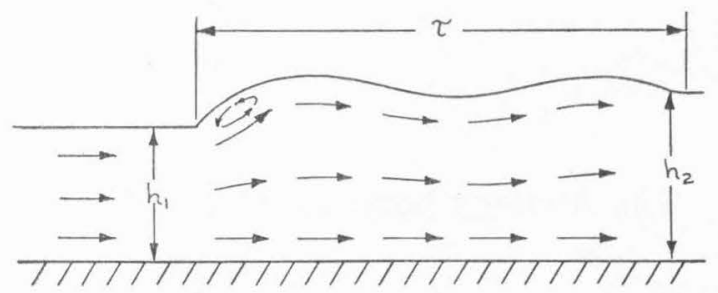

FIG. 3. Typical profile of a weak hydraulic jump $\left(h_{2} / h_{1}<2\right)$.

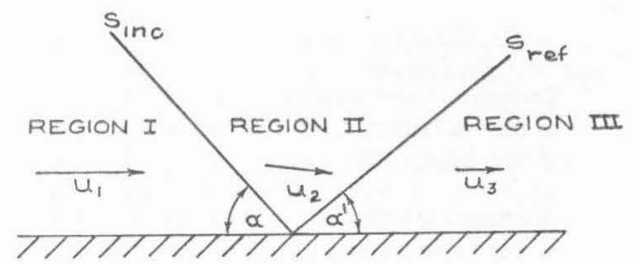

FIG. 4. Regular reflection of a shock from a rigid wall.

strong hydraulic jumps with a height ratio, $h_{2} / h_{1}$, greater than 2 have the simple profile shown in Fig. 2; in these strong hydraulic jumps, the thickness, $\tau$, of the jump is four to six times the height of the jump, $h_{2}-h_{1}$. Weaker jumps generally have the undulatory form shown in Fig. 3. The surface first rises above the final level and then oscillates with diminishing amplitude about the final level. The thickness of the undulatory jump is not well defined, but the undulations generally die out at distances of three to ten times the initial water height, $h_{1}$, the distance being greater for the smaller jumps. A theoretical explanation of this general behavior has not as yet been given.

As in the case of compression shocks in gases, flow relations across straight hydraulic jumps are determined by the conservation laws for mass, momentum, and energy without consideration of the conditions inside the jump. The result for the height and velocity ratio of a jump normal to the flow is

$$
h_{2} / h_{1}=u_{1} / u_{2}=\left(2 u_{1}^{2} / g h_{1}+\frac{1}{4}\right)^{\frac{1}{2}}-\frac{1}{4} .
$$

The height ratio given by Eq. (1) is plotted against initial Mach number as the solid curve in Fig. 1. "Mach number" in this case is defined as $u /(g h)^{\frac{1}{2}}$, since $(g h)^{\frac{1}{2}}$ is the velocity of infinitesimal surface waves.

Flow relations across oblique hydraulic jumps may be calculated in the same way as for oblique compression shocks. Similarly, flow relations across curved jumps can be found, provided the radius of curvature is much larger than the jump thickness. In many experi- 
mental situations, however, the thickness of the jump may be of the same order of magnitude as the radius of curvature. Existing theory does not cover this case.

\section{The Analogy between Shocks and Jumps}

It may be shown ${ }^{1-4}$ that the smooth free-surface flow of a liquid over a horizontal bed obeys equations of motion similar to those for the isentropic two-dimensional flow of a hypothetical gas with specific heat ratio $\gamma=2$. The mathematical correspondence is between the following quantities: the liquid velocity ratio and the gas velocity ratio; and the liquid depth ratio on the one hand and the gas density ratio, the gas temperature ratio, and the square root of the gas pressure ratio, on the other hand. It is to be noted that the isentropic condition makes the gas density ratio, the gas temperature ratio and the square root of the gas pressure ratio all equal when $\gamma=2$.

Flows with shocks or jumps are not isentropic and it might be expected that the quantitative correspondence breaks down. The entropy change, however, is of the third order in the shock strength so that weak shocks are very nearly isentropic; and the quantitative similarity should then hold to a good approximation. This situation is demonstrated in Fig. 1, which compares values of analogous quantities for the compression shock and the hydraulic jump. The curves for a gas with $\gamma=2$ approach the liquid curve asymptotically as the shock becomes weaker.

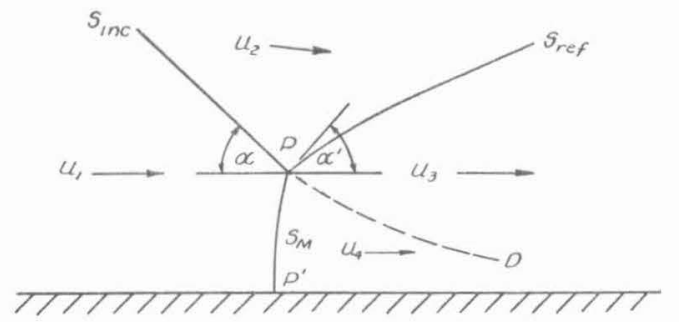

FIG. 5. Mach reflection of a shock from a rigid wall.

An analogy based on a hypothetical gas with $\gamma=2$ is somewhat unrealistic since kinetic theory shows that gases must have specific-heat ratios in the range $1<\gamma \leqslant 5 / 3$. Figure 1 includes curves for a gas with $\gamma=1.4$, which is the value for air. Although these curves do not have an asymptotic approach to the liquid curve as the shock strength is decreased, some of the flow parameters, e.g., $u_{1} / u_{2}$, have a smaller deviation over a range of strong shocks than the corresponding parameters for $\gamma=2$.

\section{Intersections and Reflections of Hydraulic Jumps and Shock Waves}

The following discussion will apply both to hydraulic jumps and compression shock waves, and the term "shock" will be used to signify either phenomenon.

Figure 4 shows the reflection of a straight shock from a plane rigid wall; it could as well refer to the intersec-

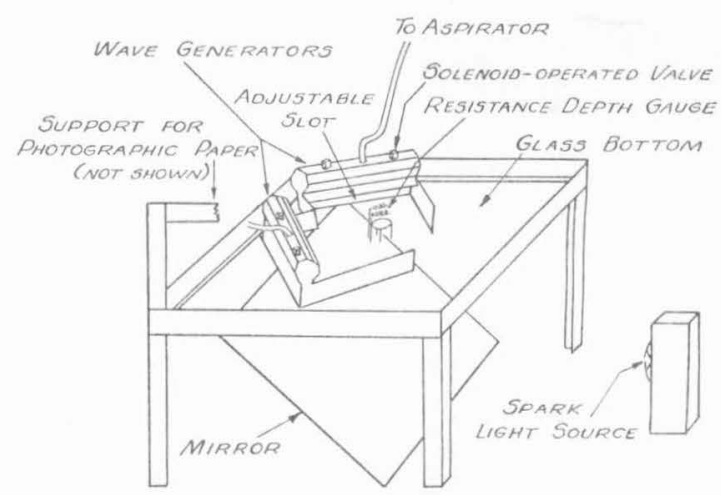

FIG. 6. A schematic representation of the experimental apparatus.

tion of two equal straight shocks where the wall becomes the plane of symmetry. The fluid in Region I, ahead of the shocks, is assumed to be either at rest or in a state of uniform motion. It is convenient to introduce a coordinate system relative to which the shocks and their intersection are stationary; in this coordinate system let $u_{1}$ be the velocity with which the fluid in Region I approaches the incident shock, $S_{\text {inc. As the }}$ flow passes through this shock, it is slowed down and deflected to a velocity $u_{2}$ in Region II. The reflected shock, $S_{\text {ref, }}$ must deflect the flow from the velocity $u_{2}$ to a velocity $u_{3}$ in Region III which, like $u_{1}$, must be parallel to the boundary wall or to the plane of symmetry. This condition is sufficient to determine the angle and strength of the reflected shock. ${ }^{11}$

When the incident shock is too strong, and when it makes too large an angle $\alpha$ with the wall, there is no real solution for the angle and strength of the reflected
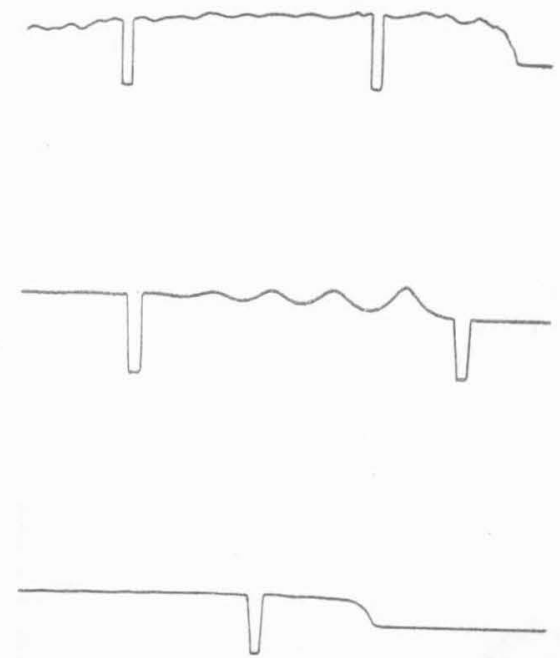

FIG. 7. Typical hydraulic-jump forms. Top curve: Strong jump with irregular roughness. Center curve: Weak jump with undulations. Bottom curve: Smooth jump of medium strength. The breaks in these records, which give the reference zero for depth, are spaced $0.6 \mathrm{sec}$. apart.

${ }^{11}$ See reference 9, pp. 318-350. 

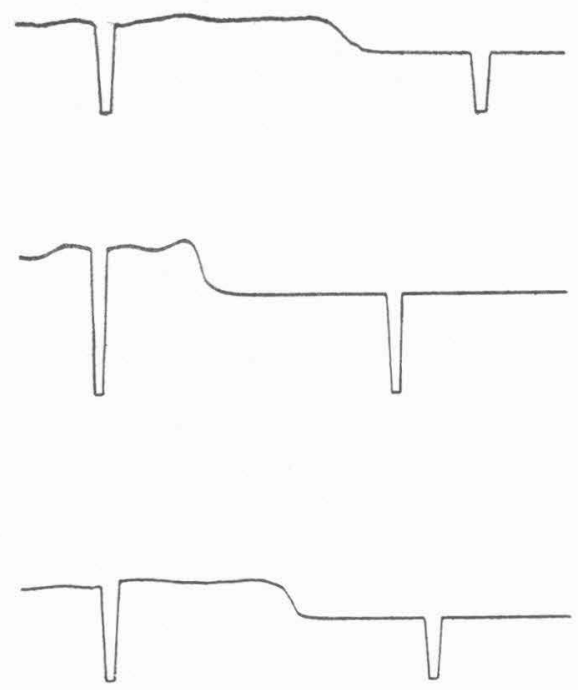

FIG. 8. Effects of surface-tension reducing agents on the jump form. Top curve: Without detergent. Center curve: Strong detergent (lauryl isoquinolinium bromide 0.1 percent). Bottom curve: Weak detergent (Kodak Photo-Flow, 0.5 percent).

shock so that the "regular" reflection scheme shown in Fig. 4 becomes impossible. In this situation the actual flow is found experimentally to follow the scheme shown in Fig. 5. A third shock, the "Mach," $S_{\mathrm{M}}$, appears, and its length, i.e., the distance $P P^{\prime}$, increases with time at a fairly constant rate. The Mach, $S_{\mathrm{M}}$, is frequently curved, and also the reflected shock, $S_{\text {ref }}$, is often curved near the triple-shock intersection.

The simplest theory of the Mach reflection assumes that all three shocks are straight. ${ }^{11}$ Coordinates are chosen fixed with respect to the shock intersection $P$ so that the problem is one of steady flow. In these coordinates, the wall (or plane of symmetry) is moving downward at a constant velocity equal to the com-

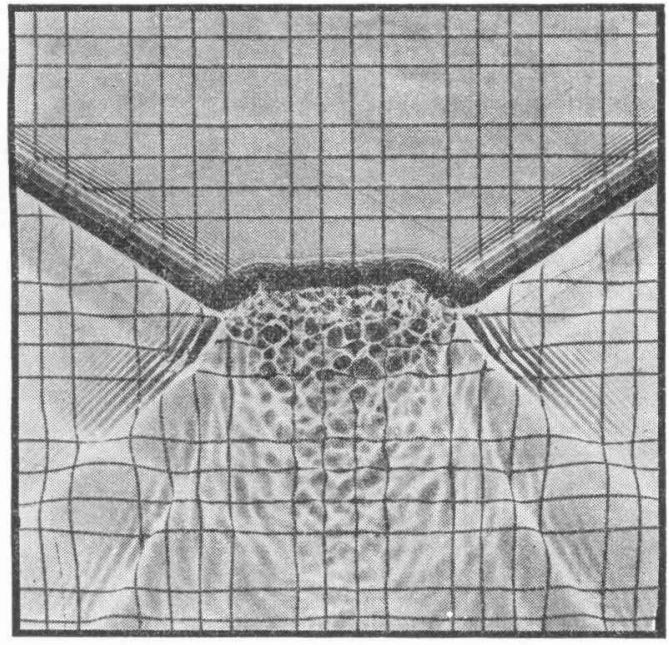

FIG. 9. Refraction photogram of medium-strength hydraulic-jump intersection $\left(\xi=0.45, \alpha=56^{\circ}\right)$. ponent of $u_{1}$ or $u_{4}$ tangential to $S_{\mathrm{M}}$. A line $P D$ can be drawn separating the fluid which has passed through $S_{\text {ine }}$ and $S_{\text {ref }}$ from that which has passed through $S_{\mathrm{M}}$. The gas pressure (or liquid height) must be continuous across $P D$ since it is not a shock. However, fluid which has passed through a single large shock has lost more flow energy than that which has passed through two smaller shocks with the same total change in pressure (or height); hence the velocity and density of a gas (or the velocity of a liquid) will be less below $P D$ than above it. Thus, $P D$ is a slip-stream. Schlieren photographs of gas flows have shown such a density discontinuity at $P D$.

Quantitative relations for such three-shock intersections can be obtained by applying the oblique shock equations to each of the shocks. The results for some ranges of interest have been tabulated by Polachek and Seeger both for compression shocks ${ }^{12}$ and for hydraulic jumps. ${ }^{13}$

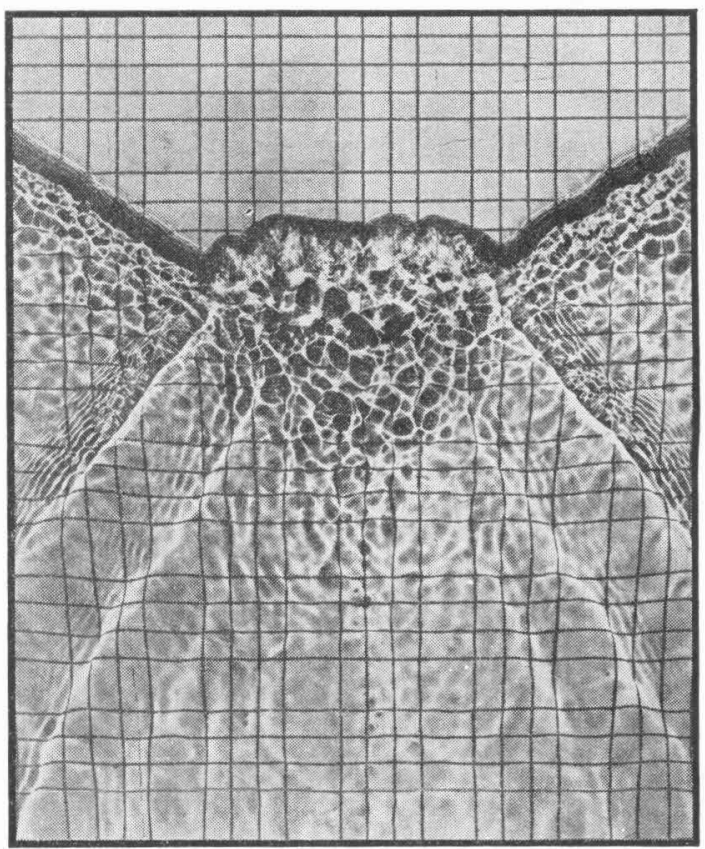

FIG. 10. Refraction photogram of strong hydraulic-jump intersection $\left(\xi=0.28, \alpha=56^{\circ}\right)$.

\section{EXPERIMENTAL MEASUREMENTS}

The purpose of the present experimental study was the investigation of regular and Mach intersections in hydraulic jumps. The apparatus used in the experiments was essentially that used by Einstein and Baird ${ }^{14}$

${ }^{12}$ H. Polachek and R. J. Seeger, Explosives Research Report No. 13, Navy Department, BuOrd, Washington, D.C. (February, 1944)

${ }^{13}$ H. Polachek and R. J. Seeger, Explosives Research Report No. 14, Navy Department, BuOrd, Washington, D.C. (August, 1944)

${ }^{14}$ H. A. Einstein and E. G. Baird, Progress Reports on the Analogy Between Surface Shock Waves on Liquids and Shocks in 


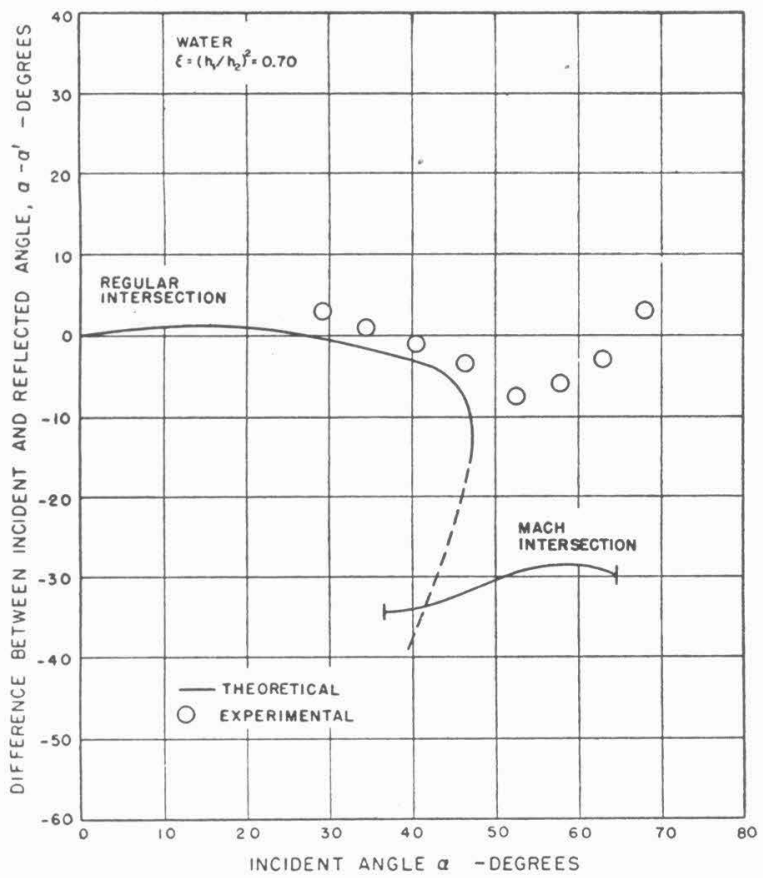

FIG. 11. Relationship between incidence and reflection angles for the intersection of weak hydraulic jumps $(\xi=0.70)$.

in earlier investigations and is shown schematically in Fig. 6. The water table was approximately $5 \mathrm{ft}$. long, $4 \mathrm{ft}$. wide, and $2 \mathrm{in}$. deep. The liquid used in the tank was a 0.001 to 0.002 normal solution of manganous chloride in distilled water; the manganous chloride was added to the water so that the electrical conductivity of the solution would be more uniform. The hydraulic jumps were produced by two wave generators which were reservoirs into which a volume of water could be raised above the free surface; this volume of water could be discharged quickly through an adjustable horizontal slot 24 in. long located just below the free surface. Instantaneous water depths at any point on the water table could be determined by measuring the resistance between a pair of partially immersed platinum electrodes. A 1000-cycle alternating voltage was applied across the electrodes and the current which flowed was recorded by an oscillograph with an upper limit of about 100 cycles on its frequency response.

Typical wave depth records are shown in Fig. 7; the record is interrupted at intervals to give a zero reference line. The horizontal coordinate in these records is a time scale not a spatial scale, but a good estimate of the instantaneous wave form in space may be obtained by multiplying this time scale by the wave velocity.

In addition to a continuous record of the water depth at a point, an instantaneous refraction photogram of the entire field could be made. The bottom of the water tank was made of glass so that the light flash from a

Compressible Gases, Hydrodynamic Laboratories, California Institute of Technology (September 15, 1946 and July 30, 1947). spark discharge across a $\frac{1}{8}$-in. gap could be transmitted upward through the water and the resulting refraction pattern could be recorded on photographic paper placed above the water surface.

\section{RESULTS AND DISCUSSION}

Measurements were first made to determine the shape and velocity of single hydraulic jumps produced with one wave generator alone; depths were determined with three pairs of electrodes. The initial water depth, $h_{1}$, ranged from about $0.5 \mathrm{~cm}$ for strong jumps to about $2 \mathrm{~cm}$ for weak jumps. Weak jumps were found to have an undulatory profile, stronger jumps had a smoother profile, and very strong jumps showed appreciable irregular roughness (cf. Fig. 7). These observations agree qualitatively with those of Bahkmeteff and Matzke ${ }^{10}$ on hydraulic jumps larger by an order of magnitude than those reported here. The velocities of the hydraulic jumps were also measured and compared with the usual formula

$$
u_{1}=\left\{g\left(h_{1}+h_{2}\right) h_{2} /\left(2 h_{1}\right)\right\}^{\frac{1}{2}},
$$

where $h_{1}$ is the water depth ahead of the jump and $h_{2}$ is the water depth behind the jump. The measured velocities of weak jumps agreed with the theoretical values within the experimental error of approximately two percent, but the measured velocities of the stronger jumps were up to five percent larger than the theoretical values. A possible explanation of this discrepancy is that the water released from the wave generator may tend to override the surface of the still water. The water

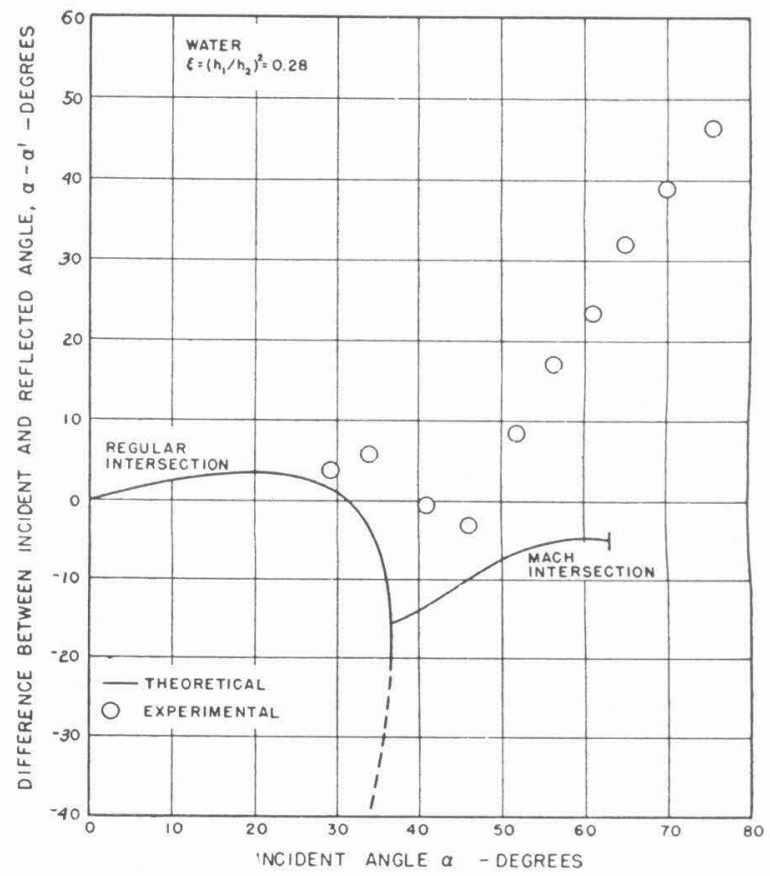

FIG. 12. Relationship between incidence and reflection angles for the intersection of strong hydraulic jumps $(\xi=0,28)$. 


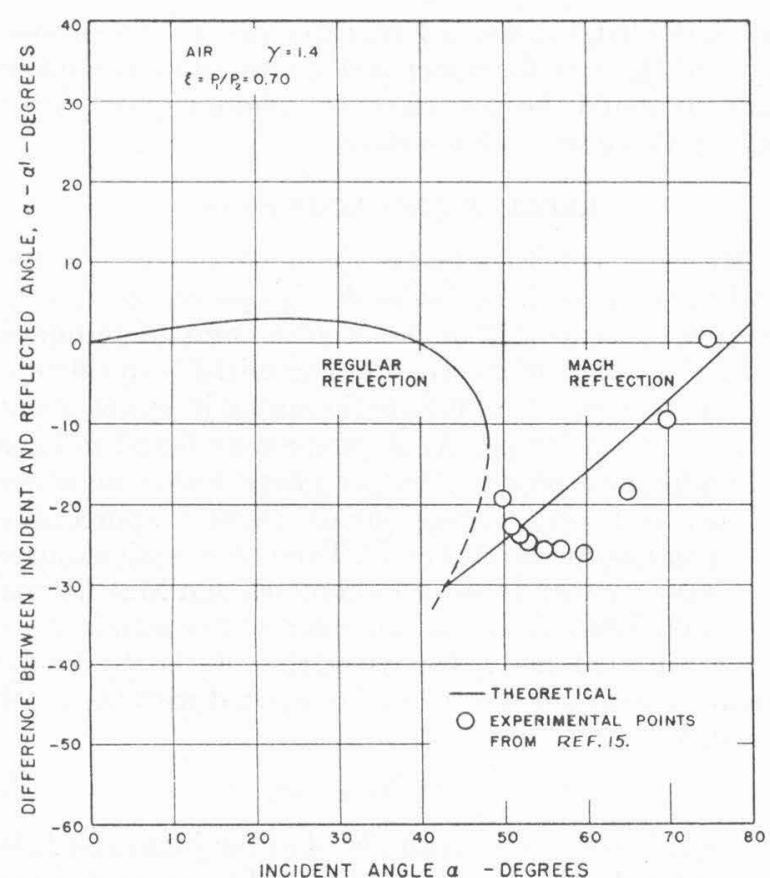

FIG. 13. Relationship between angles of incidence and reflection for the reflection of weak compression shocks $(\xi=0.70)$ in air. The experimental points are from the data of Harrison and Bleakney (see reference 15).

in the jump would then have a higher velocity at the free surface than at the bed so that the simple theory would not apply.

A study was also made of the effect of the addition of surface-tension reducing agents to the water. When 0.1 percent by volume of lauryl isoquinolinium bromide was added to the water, the wave fronts formed became steeper, and the strong jumps became smoother. However, an appreciable amount of foam was produced which interfered with the photographic procedure. It was noted, moreover, that the amplitude of the undulations in the weaker jumps was increased. A weaker detergent solution, which consisted of 0.5 percent by volume of Kodak Photo-Flo (an aerosol solution), was found to give an intermediate effect: the undulations were enhanced only in the weakest waves, while the wave form of the strong waves was smoothed and steepened. The effect of these detergent solutions is shown in Fig. 8.

Measurements were made on the hydraulic jump intersections produced by two wave generators set at various angles. The jump strengths were determined by the resistance depth gauges while the general flow pattern was recorded on the refraction photograms (cf. Figs. 9 and 10).

Most of the measurements were made with jump strengths, $\xi \equiv\left(h_{1} / h_{2}\right)^{2}$, of $0.28,0.45$, and 0.70 . For the medium and strong jumps ( $\xi=0.45$ and 0.28 ), the aerosol solution was used, while for the weak jumps $(\xi=0.70)$ detergent-free water was used, since this gave the best wave form. Figures 11 and 12 show the experimentally measured differences between the angle of incidence, $\alpha$, and the angle of reflection, $\alpha^{\prime}$, plotted against $\alpha$ for weak and strong jumps, respectively. Theoretical curves for the regular and Mach intersections are also shown. The agreement between theory and experiment is fair for the limited number of regular intersections investigated, but is definitely poor for the Mach intersections. There is little evidence of the sudden change in reflection angle which is predicted by the theory in passing from regular to Mach intersections.

These figures may be compared with Figs. 13 and 14, which give the corresponding data for intersections of compression shocks in air. The experimental points are taken from a report by Harrison and Bleakney. ${ }^{15}$ The agreement between theory and experiment is seen to be better here, but an appreciable discrepancy remains, especially for the weaker shocks. There appears to be little resemblance between the trend of the disagreement in the liquid and gas cases.

One possible error in the use of the simple theory in the above comparisons is the neglect of the curvature of the Mach jump. The geometric effect of this curvature can be eliminated by considering only a small region about the triple jump intersection. Such a presentation of the data is shown in Figs. 15 and 16 where $\omega$ and $\omega^{\prime}$ are the local angles which the incident and

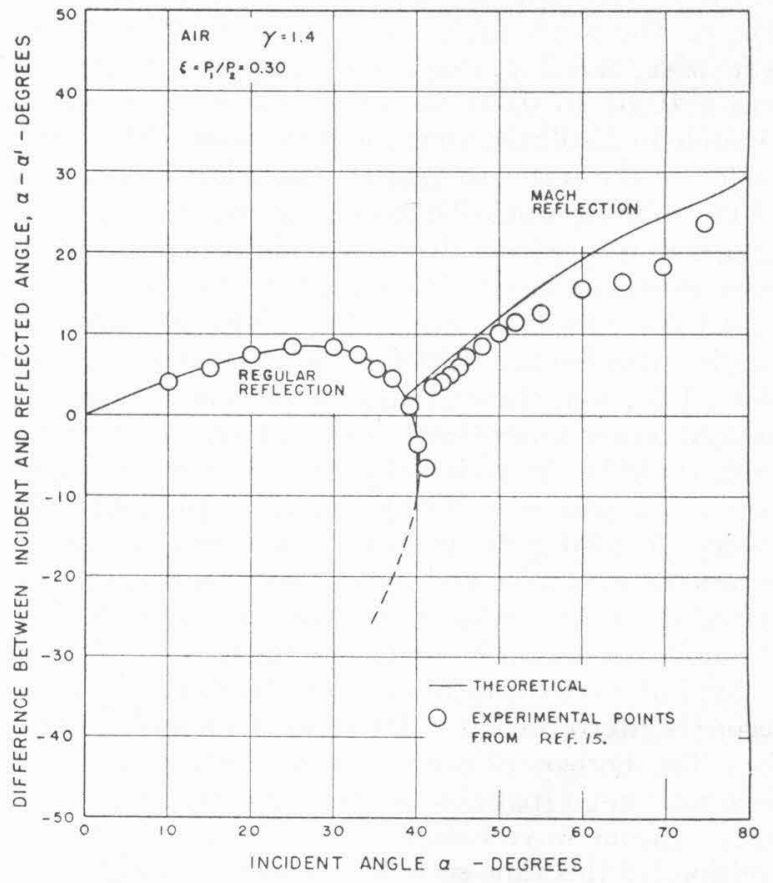

FIG. 14. Relationship between angles of incidence and reflection for the reflection of strong compression shocks $(\xi=0.30)$ in air. The experimental points are from the data of Harrison and Bleakney (see reference 15).

${ }^{15}$ F. B. Harrison and W. Bleakney, "Remeasurement of reflection angles in regular and Mach reflection of shock waves," Physics Department, Princeton University (March, 1947). 


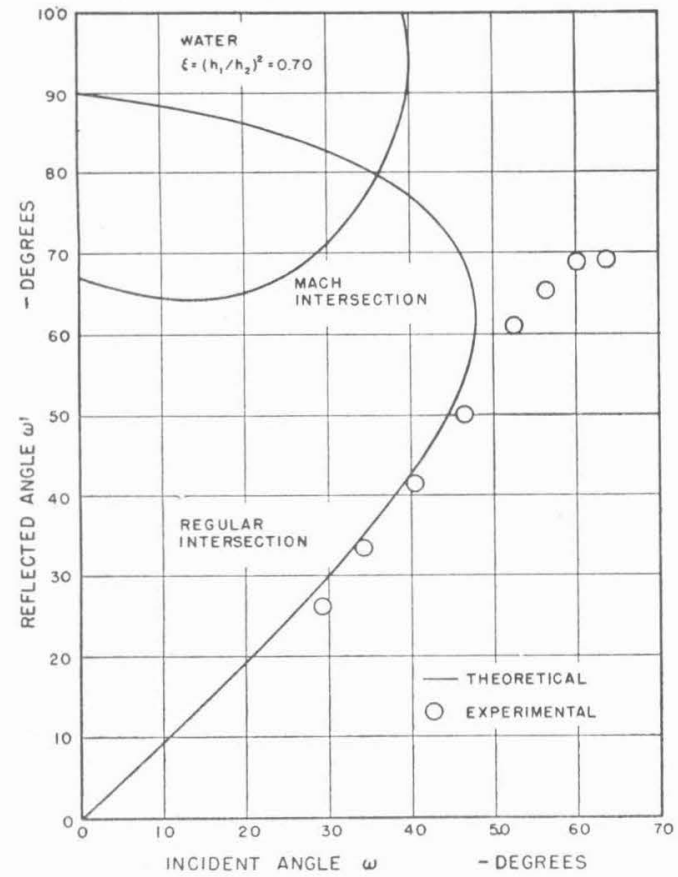

FIG. 15. Relationship between incidence and reflection angles, measured with respect to the direction of motion of the triple intersection, for the intersection of weak hydraulic jumps $(\xi=0.70)$.

reflected jumps make with the direction of motion of the triple point. The weak-jump data shown in Fig. 15 show a disagreement with theory similar to that found by Harrison and Bleakney for air, although the discrepancy is somewhat greater for the hydraulic case. The strong-jump data shown in Fig. 16 show similar disagreement with theory, whereas Harrison and Bleakney found very little discrepancy in air.

Examination of the photograms indicates that the thickness of the Mach jump is not negligible compared with the radius of curvature of the Mach. The present theory does not take account of this possibility so that an error of unknown magnitude has been introduced. This difficulty does not arise in the aerodynamic case.

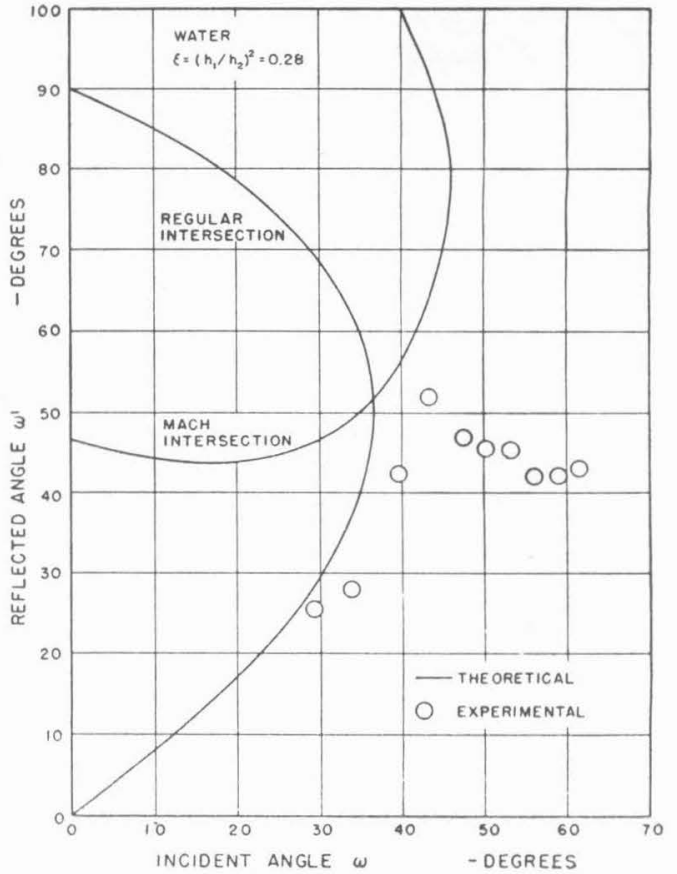

FIG. 16. Relationship between incidence and reflection angles, measured with respect to the direction of motion of the triple intersection, for the intersection of strong hydraulic jumps $(\xi=0.28)$.

The experimental observotions indicate an additional source of error in the simple theory: the liquid depth behind the reflected jump and the Mach jump is not uniform; in fact, there sometimes appears to be an additional hydraulic jump approximately parallel to, but behind, the reflected jump. Corresponding sources of error may exist in the aerodynamic case although direct observations on this possibility are not available.

One may conclude from the experimental observations discussed here that the deviations from the simple theory of hydraulic-jump intersections limit the use and validity of the hydraulic analog as a means for studying compression-shock intersections in gases. 